\title{
Decay Constants of the Heavy-Light Mesons $D^{(*)}$ and $B^{(*)}$ : Isospin Breaking
}

\section{Wolfgang Lucha*}

Institute for High Energy Physics, Austrian Academy of Sciences, Nikolsdorfergasse 18, A-1050

Vienna, Austria

E-mail: Wolfgang.Lucha@oeaw.ac.at

\section{Dmitri Melikhov}

Institute for High Energy Physics, Austrian Academy of Sciences, Nikolsdorfergasse 18, A-1050

Vienna, Austria, and

D. V. Skobeltsyn Institute of Nuclear Physics, M. V. Lomonosov Moscow State University,

119991, Moscow, Russia, and

Faculty of Physics, University of Vienna, Boltzmanngasse 5, A-1090 Vienna, Austria

E-mail: dmitri_melikhov@gmx.de

\section{Silvano Simula}

INFN, Sezione di Roma Tre, Via della Vasca Navale 84, I-00146 Roma, Italy

E-mail: simula@roma3.infn.it

\begin{abstract}
QCD sum rules are analytic predictions of hadron observables from quantum chromodynamics. In the so-called local-duality limit of their Borel-transformed form, all nonperturbative contributions are encompassed by just a single quantity, an effective threshold beyond which, by assumption, all hadronic contributions are reliably described by perturbative QCD. Reconstructing its quark-mass dependence enables us to investigate the differences of the leptonic decay constants of heavy-light mesons effectuated by the tiny isospin-violating discrepancy of the masses of up and down quarks. By and large, our findings enjoy excellent agreement with the outcomes of earlier studies, the only exception being a lattice-QCD prediction for the $B$ mesons, where we find dramatic disagreement.
\end{abstract}

The European Physical Society Conference on High Energy Physics 5-12 July 2017

Venice, Italy

\footnotetext{
*Speaker.
} 
QCD sum rules [1] relate hadron properties to the fundamental parameters of the quantum field theory of strong interactions, quantum chromodynamics; they are found from correlators of suitable interpolating operators by insertion of complete sets of hadron states and use of the operator product expansion. Applying Borel transformations removes all subtraction terms and suppresses all hadron contributions above the ground states. Any ignorance about heavier hadron states is swept under the carpet by invoking quark-hadron duality which assumes QCD and hadron contributions to mutually cancel above effective thresholds. The accuracy [2] of extracted predictions is considerably raised if taking into account such thresholds' dependence [3] on the parameters of the Borel transformations. This advanced QCD sum-rule technique led us to revisit the heavy-light-meson decay constants [4], specifically, their relative magnitude [5]. Recently, we embarked on the analysis of isospin breaking induced in heavy-light-meson decay constants by the tiny up and down quark mass difference [6,7].

In the local-duality limit of vanishing Borel variables, the QCD sum rule for the decay constant $f_{H_{q}}$ of a meson $H_{q}$ formed by a heavy quark $Q=c, b$ of mass $m_{Q}$ and a light quark $q=u, d, s$ of mass $m_{q}$ reduces to a dispersion integral of a spectral density $\rho$ found perturbatively from QCD in form of a series expansion in powers of the strong fine-structure constant $\alpha_{\mathrm{s}}$, with all nonperturbative effects subsumed by its effective threshold $s_{\text {eff }}$; the inevitable truncation of the perturbative series entails an unphysical dependence of the spectral density and the effective threshold on renormalization scales:

$$
f_{H_{q}}^{2}=\int_{\left(m_{Q}+m_{q}\right)^{2}}^{s_{\mathrm{eff}}\left(m_{Q}, m_{q}\right)} \mathrm{d} s \rho\left(s, m_{Q}, m_{q}, \alpha_{\mathrm{s}}\right) .
$$

Utilizing spectral densities up to $O\left(\alpha_{\mathrm{s}} m_{q}^{1}\right)$ and $O\left(\alpha_{\mathrm{s}}^{2} m_{q}^{0}\right)$ [8-10], we derive the decay constants $f_{P}$ and $f_{V}$ of pseudoscalar mesons $P$ and vector mesons $V$, with masses $M_{P}$ and $M_{V}$, defined in terms of the axialvector and vector currents $A_{\mu}(x)=\bar{q}(x) \gamma_{\mu} \gamma_{5} Q(x)$ and $V_{\mu}(x)=\bar{q}(x) \gamma_{\mu} Q(x)$ according to $\left\langle 0\left|A_{\mu}(0)\right| P(p)\right\rangle=\mathrm{i} f_{P} p_{\mu}$ and $\left\langle 0\left|V_{\mu}(0)\right| V(p)\right\rangle=f_{V} M_{V} \varepsilon_{\mu}(p)$. Using modified minimal subtraction renormalization for the definition of parameters optimizes [9] perturbative convergence, cf. Table 1.

Table 1: Numerical values of input parameters in the modified minimal-subtraction renormalization scheme.

\begin{tabular}{llr}
\hline Quantity & Numerical input value & Reference \\
\hline$\alpha_{\mathrm{s}}\left(M_{Z}\right)$ & $0.1182 \pm 0.0012$ & {$[11]$} \\
$\frac{1}{2}\left(m_{u}+m_{d}\right) \equiv m_{u d}(2 \mathrm{GeV})$ & $(3.70 \pm 0.17) \mathrm{MeV}$ & {$[11]$} \\
$\left(m_{d}-m_{u}\right)(2 \mathrm{GeV})$ & $(2.67 \pm 0.22) \mathrm{MeV}$ & {$[11]$} \\
$m_{s}(2 \mathrm{GeV})$ & $(93.9 \pm 1.1) \mathrm{MeV}$ & {$[11]$} \\
$m_{c}\left(m_{c}\right)$ & $(1.275 \pm 0.025) \mathrm{GeV}$ & {$[12]$} \\
$m_{b}\left(m_{b}\right)$ & $(4.247 \pm 0.034) \mathrm{GeV}$ & {$[13]$} \\
\hline
\end{tabular}

We fathom the discrepance $f_{H_{d}}-f_{H_{u}}$ of our heavy-light meson decay constants by tracking the response $f_{H}\left(m_{q}\right)$ of the local-duality QCD sum rule to continuous variations of the light-quark mass $m_{q}$ in its interval $\left[0, m_{s}\right]$. Given the perturbative spectral density at appropriately high order, the only required ingredient is the effective threshold's quark-mass dependence $s_{\text {eff }}=s_{\text {eff }}\left(m_{Q}, m_{q}\right)$. Shuffling together available knowledge on heavy-quark expansion and chiral logarithmic corrections [14], we distil parametrizations of $z_{\text {eff }} \equiv \sqrt{s_{\text {eff }}}-m_{Q}-m_{q}[15]$ the coefficients of which are fixed by matching 

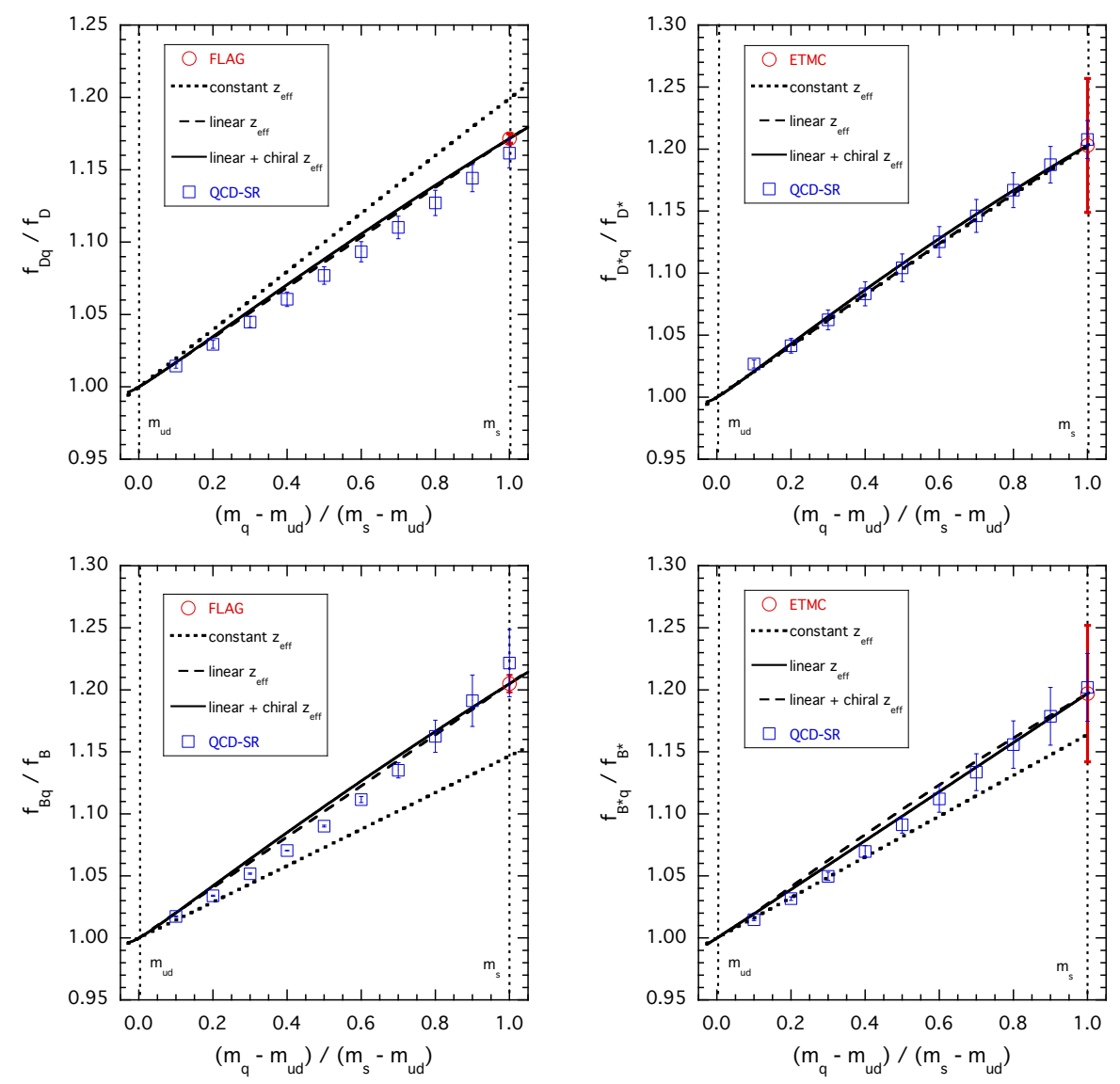

Figure 1: Behaviour of $f_{H_{q}} / f_{H}\left(m_{u d}\right)$ with redefined $m_{q}$ [15], compared with the results of Ref. [7] (squares).

$f_{H}\left(m_{q}\right)$ for $m_{q}=m_{u d} \equiv \frac{1}{2}\left(m_{u}+m_{d}\right)$ and $m_{q}=m_{s}$ to lattice-QCD results [11,16]. With respect to the $m_{q}$ dependence, we consider three ansätze for $z_{\text {eff }}$ : constant, linear and linear plus chiral logarithms. Averaging over the two latter variants within the renormalization-scale ranges $1-3 \mathrm{GeV}$ for charmed mesons and 3-6 GeV for bottom mesons converts the resulting functional behaviour $f_{H}\left(m_{q}\right)$ (Fig. 1) to our prediction for the isospin-breaking differences of the heavy-meson decay constants (Table 2).

Table 2: Local-duality isospin breaking results for heavy-meson decay constants, exhibiting good agreement with Ref. [7] (apart from the $D^{* \pm, 0}$ ) and lattice-QCD outcomes for the $D^{ \pm, 0}$ but a notable tension for the $B^{0, \pm}$.

\begin{tabular}{ccccc}
\hline & \multicolumn{4}{c}{$f_{H_{d}}-f_{H_{u}}[\mathrm{MeV}]$} \\
\cline { 2 - 5 } Mesons $H_{q}$ & Borelized threshold [7] & Local-duality limit [15] & \multicolumn{2}{c}{ Lattice QCD } \\
\hline$D^{ \pm, 0}$ & $0.97 \pm 0.13$ & $0.96 \pm 0.09$ & $0.94_{-0.12}^{+0.50}$ & {$[17]$} \\
$D^{* \pm, 0}$ & $1.73 \pm 0.27$ & $1.18 \pm 0.35$ & - & \\
$B^{0, \pm}$ & $0.90 \pm 0.13$ & $1.01 \pm 0.10$ & $3.8 \pm 1.0$ & {$[18]$} \\
$B^{* 0, \pm}$ & $0.81 \pm 0.11$ & $0.89 \pm 0.30$ & - & \\
\hline
\end{tabular}


Acknowledgement. D.M. was supported by the Austrian Science Fund (FWF), project no. P29028.

\section{References}

[1] M. A. Shifman, A. I. Vainshtein, and V. I. Zakharov, Nucl. Phys. B 147 (1979) 385.

[2] W. Lucha, D. Melikhov, and S. Simula, Phys. Rev. D 76 (2007) 036002, arXiv:0705.0470 [hep-ph]; Phys. Lett. B 657 (2007) 148, arXiv:0709.1584 [hep-ph]; Phys. Atom. Nucl. 71 (2008) 1461; Phys. Lett. B 671 (2009) 445, arXiv:0810.1920 [hep-ph]; D. Melikhov, Phys. Lett. B 671 (2009) 450, arXiv:0810.4497 [hep-ph].

[3] W. Lucha, D. Melikhov, and S. Simula, Phys. Rev. D 79 (2009) 096011, arXiv:0902.4202 [hep-ph]; J. Phys. G 37 (2010) 035003, arXiv:0905.0963 [hep-ph]; Phys. Lett. B 687 (2010) 48, arXiv:0912.5017 [hep-ph]; Phys. Atom. Nucl. 73 (2010) 1770, arXiv:1003.1463 [hep-ph]; W. Lucha, D. Melikhov, H. Sazdjian, and S. Simula, Phys. Rev. D 80 (2009) 114028, arXiv:0910.3164 [hep-ph].

[4] W. Lucha, D. Melikhov, and S. Simula, J. Phys. G 38 (2011) 105002, arXiv:1008.2698 [hep-ph]; Phys. Lett. B 701 (2011) 82, arXiv:1101.5986 [hep-ph]; Phys. Lett. B 735 (2014) 12, arXiv:1404.0293 [hep-ph]; EPJ Web Conf. 80 (2014) 00043, arXiv:1407.5512 [hep-ph].

[5] W. Lucha, D. Melikhov, and S. Simula, EPJ Web Conf. 80 (2014) 00046, arXiv:1410.6684 [hep-ph]; arXiv:1411.3890 [hep-ph]; AIP Conf. Proc. 1701 (2016) 050007, arXiv:1411.7844 [hep-ph]; Phys. Rev. D 91 (2015) 116009, arXiv:1504.03017 [hep-ph]; PoS (EPS-HEP2015) 532, arXiv:1508.07595 [hep-ph].

[6] W. Lucha, D. Melikhov, and S. Simula, EPJ Web Conf. 129 (2016) 00026, arXiv:1609.02382 [hep-ph]; EPJ Web Conf. 137 (2017) 06017, arXiv:1609.09388 [hep-ph].

[7] W. Lucha, D. Melikhov, and S. Simula, Phys. Lett. B 765 (2017) 365, arXiv:1609.05050 [hep-ph].

[8] K. G. Chetyrkin and M. Steinhauser, Phys. Lett. B 502 (2001) 104, arXiv:hep-ph/0012002; Eur. Phys. J. C 21 (2001) 319, arXiv:hep-ph/0108017.

[9] M. Jamin and B. O. Lange, Phys. Rev. D 65 (2002) 056005, arXiv:hep-ph/0108135.

[10] P. Gelhausen, A. Khodjamirian, A. A. Pivovarov, and D. Rosenthal, Phys. Rev. D 88 (2013) 014015 , arXiv:1305.5432 [hep-ph]; Phys. Rev. D 89 (2014) 099901(E); 91 (2015) 099901(E).

[11] FLAG Working Group (S. Aoki et al.), Eur. Phys. J. C 74 (2014) 2890, arXiv:1310.8555 [hep-lat]; Eur. Phys. J. C 77 (2017) 112, arXiv:1607.00299 [hep-lat].

[12] Particle Data Group (C. Patrignani et al.), Chin. Phys. C 40 (2016) 100001.

[13] W. Lucha, D. Melikhov, and S. Simula, Phys. Rev. D 88 (2013) 056011, arXiv:1305.7099 [hep-ph]; PoS (EPS-HEP 2013) 363, arXiv:1309.5611 [hep-ph].

[14] S. R. Sharpe and Y. Zhang, Phys. Rev. D 53 (1996) 5125, arXiv:hep-lat/9510037.

[15] W. Lucha, D. Melikhov, and S. Simula, preprint HEPHY-PUB 982/17, arXiv:1702.07537 [hep-ph].

[16] V. Lubicz, A. Melis, and S. Simula, PoS (LATTICE2016) 291, arXiv:1610.09671 [hep-lat].

[17] Fermilab Lattice and MILC Collaborations (A. Bazavov et al.), Phys. Rev. D 90 (2014) 074509, arXiv:1407.3772 [hep-lat].

[18] HPQCD Collaboration (R. J. Dowdall et al.), Phys. Rev. Lett. 110 (2013) 222003, arXiv:1302.2644 [hep-lat]; J. L. Rosner, S. Stone, and R. S. Van der Water, arXiv:1509.02220 [hep-ph], published in Ref. [12]. 